\title{
Approaches to Analyzing Experiments with Factorial Arrangements of Treatments Plus Other Treatments
}

\author{
Richard P. Marini \\ Department of Horticulture, Virginia Polytechnic and State University, \\ Blacksburg, VA 24061-0321
}

Additional index words. analysis of variance, contrasts, statistics

\begin{abstract}
Experiments with factorial arrangements of treatments plus one or more other treatment(s) are sometimes analyzed with a one-way analysis of variance (ANOVA) and means are separated with a multiple comparison. A set of single degree-of-freedom contrasts in a one-way ANOVA, provides formal tests for main effects and interactions. Data from a $2 \times 3$ factorial experiment that also contained a control were analyzed with a one-way ANOVA with a multiple comparison. Results from this analysis were compared to results obtained from a two-way ANOVA, a one-way ANOVA with pre-planned contrasts, a twoway ANOVA with least squares means comparisons obtained with SAS/general linear models procedure, and a regression model with an indicator variable and random blocks obtained with SAS/Mixed procedure. Results and interpretation differed depending on how the data were analyzed and these differences are discussed.
\end{abstract}

Plant biologists often use factorial experiments to study the effect of two or more factors on some aspect of plant growth and development. Aset of factorial treatments, consisting of combinations of at least two levels of two or more factors, has a well-defined structure. In a complete factorial experiment, every level of one factor is combined with every level of the other factor(s) resulting in $\mathrm{n}_{\mathrm{a}} \times \mathrm{n}_{\mathrm{b}} \times \mathrm{n}_{\mathrm{c}}$ treatment combinations, where $n$ is the number of levels of factor $A, n_{b}$ is the number of levels of factor $B$, and $n_{c}$ is the number of levels of factor C. Data from experiments with factorial structure are usually subjected to a multi-way analysis of variance (ANOVA) to evaluate each factor (main effects) and the interaction(s) of the factors.

Researchers sometimes perform experiments with factorial treatments, but also include a control or other relevant treatment(s) that do not fit the factorial structure. Experiments having a complete factorial set of treatments plus one or more additional treatments are sometimes called "augmented factorials" (Lentner and Bishop, 1993) or "factorial plus" (Lentner and Bishop, 1993). An example would be an experiment involving transplanting nursery trees. Trees in a nursery are dug and transplanted either in the fall or the spring and they are removed from the nursery with a small or a large root ball. This $2 \times 2$ factorial is then augmented with a nontransplanted control, making five treatments in the experiment. There are several situations where augmented factorials may be desirable: 1) where funding, labor, or plant material is limited;2) when some treatment combinations are not of interest; and 3) if two sets of treatments are included in an experiment, one set may be a factorial arrangement, but the other set need not be (Federer, 1955).

Received for publication 26 Sept. 2001. Accepted for publication 26 Apr. 2002.
Data from experiments with augmented factorial structure are sometimes analyzed with a one-way analysis of variance (ANOVA) and means of treatment combinations are separated with post hoc multiple comparison tests such as least significant difference (LSD) (Grey et al., 2001), Waller-Duncan LSD (Stover et al., 2001), or Tukey's honestly significant difference (HSD) (Sullivan and Pasian, 2000). Sometimes only the factorial portion of the experiment is included in the multi-way ANOVA and the mean for the extra treatment is presented in the table for comparative purposes (Lester and Grusak, 2001). Much information is lost with such an approach because the factorial components (main effects and interactions) are not investigated. Multiple comparisons are typically used with nonstructured treatments, but when means have structure more specific comparisons can be constructed depending on the treatment structure and research objectives of the particular experiment (Chew, 1977; Lentner and Bishop, 1993). Additionally, erroneous conclusions can be drawn when interactions are ignored. Augmented factorial experiments actually have two groups of treatments: 1) those of the complete factorial; and 2) the other treatments that are added to the factorial. The factorial part of the experiment can be evaluated by partitioning the treatment sums of squares into components by contrasts to provide information about the main effects and interactions(s). Other contrasts can be formed to compare two groups, such as the control vs. all factorial treatments.

Many statistics textbooks discuss orthogonal contrasts. A set of contrasts is considered orthogonal if the comparisons are independent (unrelated) of each other and the sums of squares for each contrast add to the treatment sums of squares. For these reasons orthogonality is desirable, but not essential (Chew, 1977). In many experiments a specific set of preplanned nonorthogonal con- trasts, dictated by the underlying structure of the data, can be developed. Comparisons suggested by data snooping should be tested with a multiple comparison procedure (Steele and Torrie, 1980).

The purpose of this paper is to compare several approaches for evaluating data from factorial experiments that contain additional treatments. For illustration purposes, unpublished data from an experiment comparing three formulations of gibberellins plus a nontreated control on apple (Malus $\times$ domestica Borkh.) fruit set were used.

\section{Materials and Methods}

An experiment was performed to evaluate the effect of three gibberellin formulations (ABG-3035,ABG-3063, and ABG-3068) each at two concentrations $\left(10\right.$ and $\left.20 \mathrm{mg} \cdot \mathrm{L}^{-1}\right)$ on fruit set of 'Sundale Spur Golden Delicious'. A water control was also included. Seven uniform limbs on each of six trees were randomly assigned to each of the treatments. Limbs were sprayed a total of four times to runoff with a hand-held sprayer at 10,19, 29, and $38 \mathrm{~d}$ after full bloom. Fruit set was calculated as the number of fruit per limb $65 \mathrm{~d}$ after bloom divided by the number of flower clusters per limb. The experimental design was a randomized complete block and trees served as blocks. The treatment structure was an augmented factorial; there was a set of factorial treatments ( 3 formulations $\times 2$ concentrations) plus a control. For comparative purposes, four types of statistical analyses were performed using SAS's general linearmodels (GLM) (SAS Institute, 1998) or Mixed procedure (SAS Institute, 1992) The SAS code for each analysis is presented in Appendix Tables 1-4.

Model 1. A one-way ANOVA was performed with the GLM procedure and the seven treatment means were compared with the LSD.

Model 2. A two-way ANOVA was performed with the GLM procedure to test main effects and their interaction. The seven least squares means for the interaction (simple effects) were compared with the probability of the difference, but main effect means were not estimable.

Model 3. A one-way ANOVA was performed with the GLM procedure. Single degree-of-freedom contrasts were used to partition treatment sums of squares to compare specific treatments or groups of treatments as well as interactions of interest. Estimate statements were used to estimate the differences between means of specific treatments or groups of main treatments of interest and to test the hypothesis that the differences were equal to zero.

Model 4. A regression-based model was developed with the Mixed procedure, where block was considered a random effect, concentration was included as a quantitative variable, and formulation was included as a class variable (also known as an indicator variable, a dummy variable, or a qualitative variable). The formulation $\times$ concentration interaction term was included to test the hypothesis that 
all three slopes are equal. The "solution and htype $=1$ " options were included in the model statement to request Type I (sequential) tests and to generate the solution vector for fixed effects. Parameter estimates from the solution vector can be used to estimate regression coefficients (Littell et al., 1996).

\section{Results}

Model 1. The one-way ANOVA (Table 1) indicates that treatment had a significant effect on fruit set $(P=0.003)$. The pair-wise mean comparison method, LSD, separated the treatments into three groups (Table 2). Treatments with the highest fruit set included ABG-35 and ABG-63 at $20 \mathrm{mg} \cdot \mathrm{L}^{-1}$ and ABG-68 at $10 \mathrm{mg} \cdot \mathrm{L}^{-1}$. The control and ABG-35 at $10 \mathrm{mg} \cdot \mathrm{L}^{-1}$ had the lowest fruit set. Other treat-

Table 1. Results from a one-way analysis of variance for an experiment with a factorial arrangement of treatments ( 3 formulations $\times 2$ concentrations) plus a control. For this analysis, each combination of formulation and concentration was considered as a treatment. ${ }^{2}$

\begin{tabular}{lrrrcr}
\hline Source & df & \multicolumn{1}{c}{ SS } & MS & F & $P>$ F \\
\hline Total & 41 & 44070 & & & \\
Block & 5 & 29172 & & & \\
Treatment & 6 & 6938 & 1156 & 4.36 & 0.0028 \\
Error & 30 & 7953 & 265 & & \\
\hline
\end{tabular}

${ }^{2}$ SAS statements to generate this analysis and the mean separation in Table 2 are presented in Appendix Table 1.

Table 2. The effect of three formulations of gibberellins at two concentrations on fruit set (fruit per 100 flower clusters). Means were obtained from the one-way analysis of variance in Table 1.

\begin{tabular}{lcc}
\hline Formulation & $\begin{array}{c}\text { Concentration } \\
\left(\mathrm{mg} \cdot \mathrm{L}^{-1}\right)\end{array}$ & $\begin{array}{c}\text { Fruit/100 } \\
\text { clusters }\end{array}$ \\
\hline Control & --- & $35.7 \mathrm{c}^{2}$ \\
ABG-35 & 10 & $50.1 \mathrm{bc}$ \\
& 20 & $66.9 \mathrm{ab}$ \\
ABG-63 & 10 & $55.2 \mathrm{~b}$ \\
& 20 & $80.3 \mathrm{a}$ \\
ABG-68 & 10 & $62.3 \mathrm{ab}$ \\
& 20 & $58.9 \mathrm{~b}$ \\
LSD & --- & 19.2 \\
\hline
\end{tabular}

${ }^{2}$ Means followed by the same letter are not different at the 5\% level of significance, by LSD. ments had intermediate fruit set. Although this analysis includes no formal test for interaction there appears to be an interaction between formulation and concentration for ABG-35 and ABG-63 vs. ABG-68 because unlike the other formulations fruit set for ABG-68 was negatively related to concentration.

Model 2. The two-way ANOVA indicates that fruit set was influenced by concentration, but not formulation, and the interaction was not significant $(P=0.1062)$ (Table 3$)$. Although the Type I sums of squares for the two-way model [formulation + concentration + (formulation $\times$ concentration)] are equal to the treatment sums of squares for treatment in the one-way model (Type I SS = 44076 with $6 \mathrm{df}$ ), the Type III sums of squares (with $5 \mathrm{df}$ ) are quite different (Tables 1 and 3 ) because of the incomplete factorial structure. In fact, the Type III sums of squares for formulation are actually incorrect because it excluded the control treatment effect. Least squares means were non-estimable for the main effects of formulation and concentration, but least squares means for simple effects means were compared with the probability of the difference (Table 4). The two multiple comparisons (Tables 2 and 4) are similar except that ABG-68 at $20 \mathrm{mg} \cdot \mathrm{L}^{-1}$ did not differ from $A B G-63$ at $20 \mathrm{mg} \cdot \mathrm{L}^{-1}$ with the two-way ANOVA (Table 4).

Model 3. The one-way ANOVA with contrasts allows one to compare specific treatments of interest (Table 5). The first contrast

Table 3. Results from a two-way analysis of variance for an experiment with a factorial arrangement of treatments ( 3 formulations $\times 2$ concentrations) plus a control, with tests for main effects and interaction. $^{2}$

\begin{tabular}{lrrrcr}
\hline Source & df & Sype III & MS & F & $P>$ F \\
\hline Total & 41 & 44076 & & & \\
Block & 5 & 29186 & & & \\
Formulation & 2 & 559 & 279 & 1.05 & 0.3613 \\
Concentration & 1 & 1475 & 1475 & 5.56 & 0.0251 \\
Interaction & 2 & 1282 & 641 & 2.42 & 0.1062 \\
\hline
\end{tabular}

${ }^{z}$ SAS statements to generate this analysis and the comparison of least squares means in Table 4 are presented in Appendix Table 2. indicates that the control is different than the average of all other treatments. The next two contrasts indicate that ABG-35 does not differ from ABG-63 and the average of ABG-35 + ABG-63 does not differ from ABG-68. Taken together, these three contrasts provide fairly convincing evidence that the three formulations influenced fruit set similarly, but compared to the control they caused increased fruit set. The fourth contrast indicates that fruit set is different for the two concentrations. The fifth contrast indicates that the concentration effect is similar for $\mathrm{ABG}-35$ and $\mathrm{ABG}-63$. The last contrast indicates that the effect of concentration on fruit set differs for the average of ABG-35 + ABG-63 compared to ABG-68. Estimate values in Table 6 are the differences between the means corresponding to the contrasts in Table 5. For example, when fruit set was averaged for all six $A B G$ treatments, the number of fruit set per 100 clusters was 26.5 greater than for the control and this value differed significantly from zero $(P=0.0009)$. Values for $\mathrm{P}>\mathrm{F}$ in Table 5 are equal to values for $P>t$ in Table 6 , but the estimate values in Table 6 allow one to evaluate the magnitude and the direction (plus or minus) of differences between the means of treatments or groups of treatments tested with the contrasts.

Model 4. Results from the regression model with an indicator variable using Type I tests performed with the MIXED procedure (Table 7) differ from results obtained from the

Table 4. Least squares means for simple effects in the interaction obtained from the two-way analysis of variance in Table 3. Least squares means for the main effects of formulation and concentration are nonestimable.

\begin{tabular}{lcc}
\hline Formulation & $\begin{array}{c}\text { Concn } \\
\left(\mathrm{mg} \cdot \mathrm{L}^{-1}\right)\end{array}$ & $\begin{array}{c}\text { Fruit/100 } \\
\text { clusters }\end{array}$ \\
\hline Control & --- & $35.7 \mathrm{c}^{2}$ \\
ABG-35 & 10 & $50.1 \mathrm{bc}$ \\
& 20 & $66.9 \mathrm{ab}$ \\
ABG-63 & 10 & $55.2 \mathrm{~b}$ \\
& 20 & $80.3 \mathrm{a}$ \\
ABG-68 & 10 & $62.3 \mathrm{ab}$ \\
& 20 & $58.9 \mathrm{ab}$ \\
\hline
\end{tabular}

${ }^{\mathrm{z}}$ Means followed by the same letter are not different at the $5 \%$ level of significance, by probability of the difference.
Appendix Table 1. SAS code for a one-way analysis of variance for an experiment with a factorial arrangement of treatments ( 3 formulations $x$ 2 concentrations) plus a control in a randomized complete-block design using the GLM procedure. Data steps were used to create a new variable (TRT). Means were separated with the least significant difference. Output is presented in Tables $1 \& 2$

DATA FRUITSET;

INPUT BLOCK FORM CONC SET;

/* The following statement creates a new variable called TRT for one-way ANOVA*/

trt=compress(form II',' II conc);

CARDS;

PROC GLM;

CLASS BLOCK TRT;

MODEL SET = BLOCK TRT;

MEANS TRT / LSD;

RUN;
Appendix Table 2. SAS code for a two-way analysis of variance for an experiment with a factorial arrangement of treatments (3 formulations $\times$ 2 concentrations) plus a control in a randomized complete-block design, using the GLM procedure. Least squares means were requested for main effects and the interaction; the multiple comparisons requested is the least significant difference method using the $P$-value approach. Output is presented in Tables 3 \& 4 .

DATA FRUITSET;

INPUT BLOCK FORM CONC SET;

CARDS;

PROC GLM

CLASS BLOCK FORM CONC;

MODEL SET = BLOCK FORM CONC FORM $*$ CONC;

LSMEANS FORM CONC FORM*CONC / PDIFF;

RUN; 
Table 5. Results from a one-way analysis of variance for an experiment with a factorial arrangement of treatments ( 3 formulations $\times 2$ concentrations) plus a control. Single degree-of-freedom contrasts are used to partition sums of squares for the main effects of formulations and concentration and to evaluate interaction. ${ }^{\mathrm{z}}$

\begin{tabular}{lrrrrr}
\hline Source & df & \multicolumn{1}{c}{ SS } & MS & F & $P>$ F \\
\hline Total & 41 & 44076 & & & \\
Block & 5 & 29186 & & & \\
Treatment & 6 & 6938 & 1156 & 4.36 & 0.0028 \\
& & Contrasts & & & \\
Control vs. all others & 1 & 3622 & 3622 & 13.66 & 0.0009 \\
main effect (35 vs. 63) & 1 & 509 & 509 & 1.92 & 0.1762 \\
main effect (35 + 63 vs. 68) & 1 & 50 & 50 & 0.19 & 0.6677 \\
concentration (10 vs. 20) & 1 & 1475 & 1475 & 5.56 & 0.0251 \\
interaction (35 $\times 63)$ & 1 & 104 & 104 & 0.39 & 0.5363 \\
interaction (35 + 63 $\times$ 68) & 1 & 1179 & 1179 & 4.45 & 0.0435 \\
\hline
\end{tabular}

${ }^{\mathrm{z}} \mathrm{SAS}$ statements to generate this analysis are presented in Appendix Table 3.

Table 6. Comparison of main effects means for formulation and concentration obtained with the Estimate statement. Estimate statements are used to average the simple effects estimates (formulation and concentration) to estimate the difference between the simple effect means. ${ }^{\mathrm{z}}$

\begin{tabular}{lccrr}
\hline Parameter & Estimate & Standard error & T-value & $P>\mathrm{t}$ \\
\hline Control vs. all others & 26.5 & 7.2 & 3.70 & 0.0009 \\
Main effect (35 vs. 63) & -9.2 & 6.6 & -1.39 & 0.1762 \\
Main effect (35 + 63 vs. 68) & 2.5 & 5.8 & 0.43 & 0.6677 \\
Concentration (10 vs. 20) & -38.4 & 16.3 & -2.36 & 0.0251 \\
Interaction $(35 \times 63)$ & 4.2 & 6.6 & 0.63 & 0.5363 \\
Interaction $(35+63 \times$. 68) & -12.1 & 5.76 & -2.11 & 0.0435 \\
\hline
\end{tabular}

${ }^{2}$ SAS statements to generate this analysis, along with coefficients for contrasts, are presented in Appendix Table 3.

two-way ANOVA performed with the GLM procedure using Type III SS (Table 3). Both analyses provided the same $P$ - values for the main effect of concentration and the interaction of formulation $\times$ concentration, but Type I test results from the MIXED procedure indicate that formulations differ $(P=0.0049)$ whereas the Type III tests of GLM procedure indicated that formulation was nonsignificant $(P=0.3613)$. The discrepancy occurred because the GLM Type III SS excluded the control treatment effect and calculated the sums of squares for formulation incorrectly. Performing a two-way
ANOVA using the Type III SS with the GLM procedure in such situations would lead one to incorrectly conclude that fruit set was similar for the three formulations. The $P$ - value for interaction, is not significant at the 5\% level, indicating that there is not enough evidence to reject the hypothesis that slopes for all three formulations are equal. However it may be informative to calculate the slopes for the three formulations. Using the estimates in the solution vector, the slopes for ABG-35, ABG63, and ABG-68 are 1.67, 2.50, and -0.34 , respectively. The slopes between ABG- 63 and
ABG-68 were significantly $(P<0.05)$ different.

\section{Discussion}

The data from this experiment would be interpreted differently depending on which statistical analysis is performed. The reason factorial experiments are performed is to evaluate a possible interaction between two factors; otherwise it would be much simpler to evaluate only one level of each factor. A one-way ANOVA, followed by a multiple comparison, does not provide formal tests for main effects or interaction(s). Although the analysis is a little more involved, a two-way ANOVA allows one to evaluate main effects and the interaction(s). However, as demonstrated in Table 3, the Type III SS in the GLM procedure may not generate appropriate tests when the factorial structure in a mixed effects model is unbalanced. Had there been three or more concentrations of each formulation, orthogonal polynomials could have been used to evaluate the dose response (linear and quadratic) for each formulation and the interaction between formulation and concentration (Steele and Torrie, 1980).

Contrasts are very powerful; sometimes treatment differences or interactions are declared significant with contrasts when the ANOVA fails to reject the hypothesis that all treatments are equal or that factors do not interact. Steele and Torrie (1980) explained that an F-test, with more than one degree-of-freedom for the numerator mean squares is an average test of as many independent comparisons as there are degrees of freedom. If only one of the comparisons involves a real difference and if this difference should be averaged with a number of non-real differences, then a test of this average might fail to detect the real difference. This was the case for interaction in Tables 3 and 7. A significant main effect and a
Appendix Table 3. SAS code for a one-way analysis of variance for an experiment with a factorial arrangement of treatments ( 3 formulations $\times 2$ concentrations) plus a control in a randomized complete-block design, using the GLM procedure. Data steps were used to create a new variable (TRT). Six single-degree-of-freedom contrasts were used to partition the treatment sums of squares. Differences between means were estimated and tested with ESTIMATE statements. Output is presented in Tables $5 \& 6$

DATA FRUITSET;

INPUT BLOCK FORM CONC SET;

/* The following statement creates a new variable called TRT for one-way ANOVA*/

trt=compress(form II',' II conc);

CARDS;

PROC GLM;

CLASS BLOCK TRT;

MODEL SET = BLOCK TRT;

* FORMULATIONS - - - - - - - - - CK 3535636368 68;

* CONCENTRATIONS - - _ - - - - 01020102010 20;

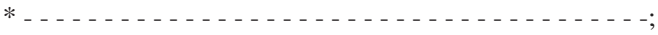

CONTRAST 'CK vs. OTHERS' TRT -6 111111111 ;

CONTRAST ' 35 vs. 63' TRT $011-1-100$;

CONTRAST ' $35+63$ vs. 68' TRT 0111111 -2 -2 ;

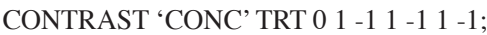

CONTRAST 'INT 35 x 63' TRT $01-1-1100$;

CONTRAST 'INT 35+63 X 68' TRT 0 1 $1-1$ 1 1 -1 -2 2 2;
ESTIMATE 'CK vs. OTHERS' TRT -1 1667.1667 .1667.1667 .1667.1667; ESTIMATE' '35 vs. 63' TRT 0.5 .5 - .5 - .500 ;

ESTIMATE' ' $35+63$ vs. 68 ' TRT 0 . 25 .25 .25 .25 - .5 - -.5 ;

estimate 'conc' trt $011-1 \quad 1-1 \quad 1-1$;

ESTIMATE 'INT 35 vs.68' TRT $0.5-.5-.5 .500$;

ESTIMATE 'INT $35+63 \times 68$ ' TRT $0.25-.25 .25-.25-.5$. 0 ; RUN;

Appendix Table 4. SAS code for a two-way analysis of variance for an experiment with a factorial arrangement of treatments (3 qualitative formulations $\times 2$ quantitative concentrations) plus a control with random blocks, using the Mixed procedure. The solution option and HTYPE $=1$ are included in the model statement to request the solution vector and TYPE I tests respectively. Output is presented in Tables $7 \& 8$.

DATA FRUITSET;

INPUT BLOCK FORM CONC SET;

CARDS;

PROC MIXED;

CLASS BLOCK FORM;

MODEL SET=FORM CONC FORM*CONC / SOLUTION HTYPE=1; RANDOM BLOCK;

RUN; 
Table 7. Results from a two-way analysis of variance, using SAS/STAT MIXED procedure, for an experiment with a factorial arrangement of treatments ( 3 qualitative formulations $\times 2$ quantitative concentrations) plus a control. ${ }^{z}$

\begin{tabular}{|c|c|c|c|c|}
\hline \multirow[b]{2}{*}{ Effect } & \multicolumn{2}{|c|}{$\mathrm{df}$} & \multirow[b]{2}{*}{$\mathrm{F}$ value } & \multirow[b]{2}{*}{$P>\mathrm{F}$} \\
\hline & Numerator & Denominator & & \\
\hline Formulation & 3 & 30 & 5.28 & 0.0049 \\
\hline Concentration & 1 & 30 & 5.56 & 0.0251 \\
\hline Interaction & 2 & 30 & 2.42 & 0.1062 \\
\hline
\end{tabular}

${ }^{2}$ SAS statements used to generate this analysis are presented in Appendix Table 4.

Table 8 . The solution vector obtained with the analysis of variance in Table 7 . The estimates can be used to calculate slopes for each formulation. ${ }^{2}$

\begin{tabular}{lcccccc}
\hline Effect & Formulation & Estimate & Standard error & df & T-value & $P>\mathrm{t}$ \\
\hline Intercept & & 65.7 & 18.8 & 5 & 3.49 & 0.0174 \\
Formulation & 0 & -29.9 & 16.3 & 30 & -1.84 & 0.0758 \\
Formulation & 35 & -32.3 & 21.0 & 30 & -1.54 & 0.1351 \\
Formulation & 63 & -32.3 & 21.0 & 30 & -1.69 & 0.1012 \\
Formulation & 68 & 0 & --- & --- & --- & --- \\
Concentration & & -0.3 & 0.9 & 30 & -0.36 & 0.7214 \\
Form. $\times$ Conc. & 0 & 0 & --- & --- & --- & --- \\
Form. $\times$ Conc. & 35 & 2.0 & 1.3 & 30 & 1.51 & 0.1407 \\
Form. $\times$ Conc. & 63 & 2.8 & 1.3 & 30 & 2.14 & 0.0407 \\
Form. $\times$ Conc. & 68 & 0 & --- & --- & --- & --- \\
\hline
\end{tabular}

${ }^{2}$ SAS statements used to generate this analysis are presented in Appendix Table 4.

significant interaction from the ANOVA are not required to perform pre-planned contrasts (Lentner and Bishop, 1993). Most experimental design texts discuss contrasts, explain how to calculate coefficients for contrasts, and provide coefficients for orthogonal polynomials.

A discussion by Littell et al. (1991) illustrates the flexibility of using contrasts as a tool for statistical analyses. They suggested using multiple comparisons to compare means when the investigator has no knowledge that might suggest specific comparisons among the treatment means. If the investigator knows something about the treatments, then specific comparisons can be planned while designing the experiment. They suggested three general types of comparisons; the most appropriate type depends on how factors might interact. The first type is called a "simple effect" comparison (Steele and Torrie, 1980). In this study it would be appropriate if the comparisons for formulation were generally different for concentration, that is, if the comparison for formulation interacts with concentration. In this study the fifth and sixth contrasts are simple effect comparisons (Table 5). The second type, estimating the comparison for formulations averaged across both concentrations, is called a "main effect" comparison. It would be appropriate if the comparison of formulations did not interact with concentration, that is, if the comparison had a similar value for both concentrations. In this study the first three contrasts are main effect comparisons (Table 5). The third type is a "compromise" between simple effect and main effect comparisons. It would be appropriate if there were subsets of formulations such that the comparisons did not interact with concentration within the subsets. In this study the sixth contrast is a compromise comparison (Table 5).

The last analysis, utilizing the regression approach with the Mixed procedure, probably provides the most information (Table 7). Unlike the GLM procedure, using the Type I tests in the Mixed procedure provided valid SE for treatment effects, indicating that the two main effects are significant. The lack of interaction indicates that the slopes for the three formulations are not different, and the slopes can be estimated from the solution vec- tor. Intercepts cannot be estimated because there was not a control associated with each formulation.

Complete factorial experiments are often desirable because they are easy to analyze and interpret. Incomplete factorials are sometimes preferable. In such cases the use of pre-planned contrasts, which are suggested by the treatment structure, is superior to a one-way ANOVA with a mean separation test because hypotheses about main effects and interactions can be tested.

\section{Literature Cited}

Chew, V. 1977. Comparisons among treatment means in an analysis of variance. ARS/H/6. USDA, Beltsville, Md.

Federer, W.T. 1955. Experimental design. Macmillan, New York.

Grey, T.L., D.C. Bridge, and D.S. Nesmith. 2001. Response of several transplanted pepper cultivars to variable rates and methods of application of clomazone. HortScience 36:104-106.

Lentner, M. and T. Bishop. 1993. Experimental design and analysis. $2^{\text {nd }}$ ed. Valley Book Co., Blacksburg, Va.

Lester, D.E. and M.A. Grusak. 2001. Postharvest application of chelated and nonchelated calcium dip treatments to commercially grown honeydew melons: Effects on peel attributes, tissue calcium concentration, quality, and consumer preference following storage. HortTechnology 11:561-566.

Littell, R.C., R. J. Freund, and P. C. Spector. 1991. SAS system for linear models. $3^{\text {rd }}$ ed. SAS Institute, Cary, N.C.

Littell, R.C., G.A. Milliken, W.W. Stroup, and R.D. Wolfinger. 1996. SAS system for mixed models. SAS Institute, Cary, N.C.

SAS Institute. 1992. Technical report P-229, SAS/ STAT software: Changes and enhancements, release 6.07. SAS Institute, Cary, N.C.

SAS Institute. 1998. SAS/STAT User's Guide, Version $6,4^{\text {th }}$ ed. SAS Institute, Cary, N.C.

Sullivan, K.J. and C.C. Pasian. 2000. Evaluation of two growing systems for cut snap dragon production: Tray vs. ground bed. HortScience 35:25-27.

Steele, R.G.D. and J.H. Torrie. 1980. Principles and procedures of statistics-A biomedical approach. $2^{\text {nd }}$ ed. McGraw Hill, New York.

Stover, E., M. Fargione, R.Risio, X. Yang, and T. Robinson. 2001. Fruit weight, crop load, and return bloom of 'Empire' apple following thinning with 6-benzyladenine and NAA at several phonological stages. HortScience 36: 1007-1081. 